\title{
Cervical Vestibular Evoked Myogenic Potential in Children with Auditory Neuropathy Spectrum Disorder of prelingual onset
}

\section{Original Article}

\author{
Mohamed El-Badry, Hesham Samy, Rafeek Abdelkader, Amira Fawzy
}

Audio-Vestibular Medicine Minia University, Egypt

\begin{abstract}
Objectives: Aims of the current work were to describe cervical vestibular evoked myogenic potential (c-VEMP) in children with ANSD of pre-lingual onset, and to compare c-VEMP findings to those in children with non- ANSD sensorineural (non -ANSD SNHL) of pre-lingual onset.

Methods: The study included 35 children with bilateral ANSD, and 32 children with bilateral non-ANSD SNHL of severe, severe to profound, or profound degree. Both groups of children had the onset SNHL pre-lingually. The study also included 15 control children. Mean age and SD were 6.9 years \pm 2.9 for the control children, 7.8 years \pm 3.1 for the ANSD children, and 8.8 years \pm 3.4 for the non-ANSD children. All participant children were subjected to full history taking, otological examination, hearing assessment, recording for auditory brainstem response and cochlear microphonics, Distortion product otoacoustic emission recording, and c-VEMP recording.

Results: Results showed that the vast majority of ANSD children ( $88 \%$ of ears) had intact c-VEMP response. Moreover, there was no statistically significant difference between the ANSD children and control children as regards the VEMP parameters (P1-N1 amplitude, asymmetric ratio, P1 latency, N1 latency, and inter-aural latency differences of P1 and N1). There was no statistically significant difference between ANSD with risk factors and those without risk factors as regards VEMP parameters. Results indicate that the vast majority of ANSD children with pre-lingual onset, even those with risk factors, have normal saccular function along with intact and normally functioning inferior vestibular nerve, and the other central connections responsible for the VEMP response. On the other hand, 53.1\% of ears children with non-ANSD SNHL had an absent c-VEMP response, and $46.9 \%$ of ears had intact and normal c-VEMP response. The overall results indicate that while the pathology of non-ANSD SNHL involves the saccule in more than half of children, the underlying pathology ANSD spares the saccule and its central connection.

Conclusions: Results suggest that the site of lesion in ANSD children of pre-lingual onset is restricted to the auditory system with sparing the vestibular system in the majority of children. These findings support the choice of cochlear implantation as the best line of management in children with ANSD, even in the presence of risk factors. So cochlear implantation in children with ANSD is safer than children with SNHL as there will be no fear of bilateral saccular dysfunction in the case saccular damage occurred in the surgery side as a result of the surgical procedure.
\end{abstract}

Key Words: Ansd, prelingual hearing loss, vemp.

Received: 26 November 2018, Accepted: 1 March 2019

Corresponding Author: Rafeek abdelkader, MD, Audio-Vestibular Medicine Minia University, Egypt Tel.: 01000432269, E-mail: rma1hfa2002@yahoo.com

ISSN: 2090-0740, June 2019 Vol.20, No.2

\section{INTRODUCTION}

Auditory Neuropathy Spectrum Disorder (ANSD) is frequently present in the pediatric population with a sensorineural hearing loss (SNHL), accounting between $10 \%$ and $14 \%$ of children with $\mathrm{SNHL}^{[1,2]}$. The prevalence is much higher in hearing-impaired infants with risk factors or graduated from the neonatal intensive care unit (NICU), 40 to $77 \%$ of those infants were reported to have $\operatorname{ANSD}^{[3,4]}$.

There has been a growing awareness of vestibular dysfunction in children with SNHL, in the recent years. One of the available procedures to evaluate parts of the vestibular system in children is vestibular evoked myogenic potential (VEMP). It is to be noted that multiples studies were done, including adults or old children with postlingual onset. Comparable data on young children with ANSD with prelingual onset are quite rare and for only three children ${ }^{[5]}$. Saccular dysfunction in children with non-neuropathy SNHL was proved by severalstudies ${ }^{[6-8]}$. Again, comparable data about VEMP results in children with ANSD are quite scarce ${ }^{[5]}$. Furthermore, VEMP in children with ANSD was not thoroughly described.

So the current study aimed to examine VEMP in children with ANSD to determine the integrity of the anatomical components of the vestibule-colic reflex (i.e., the saccule, inferior vestibular nerve, and the higher central connections) in children with ANSD. Then to compare 
VEMP findings to those in children with profound nonneuropathy SNHL

Another target of this research is to determine if the VEMP can separate the presynaptic site of the lesion from the postsynaptic site of lesion in ANSD. This target was addressed by comparing VEMP findings in ANSD children without risk factors (assuming to have presynaptic ANSD) to VEMP findings in children with risk factors (expecting to have postsynaptic ANSD). We hypothesized that, in presynaptic ANSD where the pathology is limited to IHCs /synapses, the VEMP response would be intact and normal, while in postsynaptic ANSD where it is assumed neuropathy in the auditory nerve, the VEMP would be affected due to similar neuropathy in the inferior vestibular nerve.

\section{PATIENTS AND METHODS}

The study group included sixty-seven children with congenital prelingual hearing loss; Thirty-five children had bilateral ANSD, 18 boys and 17 girls. Their age ranged between 3.9 years and 13.9 years, with a mean of 7.8 years and standard deviation (SD) of 3.0 years. The criteria to diagnose ANSD were absent or severely abnormal ABR, intact $\mathrm{CM} \&$ intact $\mathrm{OAE}$ and absent acoustic reflex ${ }^{[9]}$. Thirty-two children had the traditional bilateral SNHL (Non-ANSD). Those children were selected to have severe, severe to profound or profound degree of hearing loss; in addition to absent ABR, absent $\mathrm{CM}$ and absent OAEs. All children of the study group were recruited from the Audiology unit in Minia university hospital, Minia, Egypt. Children, less than three years were excluded because it is difficult for them to perform the VEMP testing. Similarly, children with conductive pathology as otitis media with effusion were banned to avoid the effect of conductive hearing loss on VEMP testing.

The control group included 15 children with their age and sex distribution matched with the study group. It consists of 7 boys and eight girls. Their age ranged between 4.6 years and 13.5 years, with a mean of 6.9 years and standard deviation (SD) of 2.9 years.

They had no history of perinatal, neonatal, or postnatal risk factors. They had bilateral normal hearing sensitivity, type A tympanograms and intact acoustic reflex with the normal acoustic reflex threshold; in addition to normal bilateral DPOAEs. They were selected from the children of medical staff members, and relatives were accompanying patients in the Audiology unit.

The parents of the participated children had been informed about the study aims and the detailed procedures to be used, before taking part. They gave written consent for their children participate in the study, and the ethical research committee approved all systems at Minia University
All children participated in the current work were subjected to the following, full history taking including prenatal, perinatal, neonatal, postnatal, developmental and family history\& otological examination and tympanometry and acoustic reflex testing. Free field audiometry, conditioned play audiometry or conventional audiometry was performed, according to the child age and reliability by using audiometer Madsen Astera.

ABR was performed using the intelligent hearing system (IHS) two channels evoked potential recording apparatus with Smart EP software, version 4.5. Electrode montage was high forehead to ipsilateral mastoid. The common electrode was placed on the contralateral mastoid. The stimuli were $100 \mu$ s alternating click delivered through Insert earphones at an intensity level of $90 \mathrm{~dB}$ nHL. The repetition rate of the stimuli was $31 \mathrm{p} / \mathrm{s}$. The response was filtered between 100 and $3000 \mathrm{~Hz}$, amplified 100,000 times, recorded over $10.24 \mathrm{~ms}$ time window, and 2000 sweeps were averaged for each run.

Primarily, the same ABR testing protocol described before was used to record the CM using the surface electrodes. The separate recording was done for the three click polarities, namely, alternating, rarefaction, and condensation. The $\mathrm{CM}$ was recorded at $90 \mathrm{~dB} \mathrm{nHL}$. The $\mathrm{CM}$ was considered present if the response reversed its polarity with the change of the stimulus polarity. Control runs were recorded with clamping the tube of the insert earphones. The absence of the response confirms that the response is biologic $\mathrm{CM}$ and not artifact. $\mathrm{ABR}$ and $\mathrm{CM}$ testing for each child were carried under chloral hydrate sedation.

Distortion Product Otoacoustic Emission (DPOAE) was recorded using the IHS two channels evoked potential recording apparatus with smart OAE 4.5 software. Two tones were used: $\mathrm{L} 1=65 \mathrm{~dB}(\mathrm{SPL})$ and $\mathrm{L} 2=55 \mathrm{~dB}$ SPL, while $\mathrm{f} 2 / \mathrm{fl}$ was 1.22 . The amplitude of the response of the distortion product (DP) at $2 \mathrm{f} 1-\mathrm{f} 2$ and background noise (Ns) were obtained at nine points corresponding to $\mathrm{f} 2$ frequencies of $553,783,1105,1560,2211,3125$, 4416, 6250 and $8837 \mathrm{~Hz}$. These measurements were used to build a DP-gram by displaying the DP against the $\mathrm{f} 2$ frequency. The signal-to-noise ratio (SNR) was measured $(\mathrm{SNR}=\mathrm{DP}-\mathrm{Ns})$ at each of these nine points. DPOAE was considered normal, thereby reflecting normal cochlear function, if the SNR was $>3 \mathrm{~dB}$ SPL on at least $70 \%$ of the tested frequencies.

Vestibular-evoked myogenic potential testing was performed using IHS two channel evoked potential recording apparatus with Smart EP software, version 4.5. The children were tested in the supine position. Electromyographic (EMG) activity was recorded ipsilaterally from the middle of the sternocleidomastoid muscle using a surface (active) electrode, with a reference electrode on the upper edge of the sternum and the ground 
electrode on the forehead. The respective electrodes were placed symmetrically. During each recording session, children were instructed and assisted in raising\& turning their heads towards the contralateral side from the tested ear and the sternocleidomastoid muscle under tension.

The tone burst which was used was $500 \mathrm{~Hz}$ with a two-cycle rise and fall time and plateau were used. They were presented at three cycles per second (through a Telephonic ${ }^{\circledR}$ TDH- 39 headphone) at $95 \mathrm{~dB} \mathrm{nHL}$. The EMG signal was amplified (10,000 times); the bandpass filter was $(30-1500 \mathrm{~Hz})$ and averaged after 100-200 sweeps. The window of analysis started $30 \mathrm{~ms}$ before stimulus onset and ended $70 \mathrm{~ms}$ after stimulus onset (from $-30 \mathrm{~ms}$ to $70 \mathrm{~ms}$ ). Every ear was stimulated separately and the first ear to be tested was selected randomly.

The Smart EP software accept data acquisition, only when the root mean square EMG activity was between 50 and $100 \mu \mathrm{V}$. Data acquisition was rejected when root mean square EMG activity was below $50 \mu \mathrm{V}$ or above 100 $\mu \mathrm{V}$. The level of root indicates square EMG activity was monitored and appeared on the computer screen, allowing the examiner or an assistant to give feedback response to the child and assist him to maintain constant muscle tension.

The measurement obtained for VEMP was the peak amplitude difference between the first positive peak (P1) and the first negative peak (N1). The corrected amplitude was computed by software by dividing the $\mathrm{P} 1-\mathrm{N} 1$ amplitude by the root mean square of EMG for the first 30 $\mathrm{ms}$ before stimulus onset. The $\mathrm{P} 1-\mathrm{N} 1$ amplitude asymmetry ratio (AR) was computed using the following equation: (larger corrected amplitude - smaller corrected amplitude)/ (larger corrected amplitude + smaller rectified amplitude) $\times 100,{ }^{[10]}$. In addition to amplitude measurement, P1\& N1 latencies and interaural latency difference were measured.

\section{RESULTS}

There is no history of a risk factor in children with SNHL except history of positive consanguinity in 18 children and history of the similar condition in 15 children. However in children with ANSD, there were five children were premature, four had low birth weight, and seven had hyperbilirubinemia while positive consanguinity was present at 19 children and history of the similar condition in 17 children, nearly identical to family history in SNHL.

In children with ANSD, VEMP was intact in 58 ears $(88 \%)$, Twenty-six children had bilateral intact VEMP, one had absent bilateral VEMP, and six had unilateral absent VEMP. (Fig. 1) displays such results. While in children with non-neuropathy SNHL VEMP was intact only in 30 ears $(46.9 \%)$, while it was absent in 34 ears $(53.1 \%)$. Thirteen children had bilateral intact VEMP, fifteen had absent bilateral VEMP, and four had unilateral absent VEMP. (Fig. 2) displays such results.

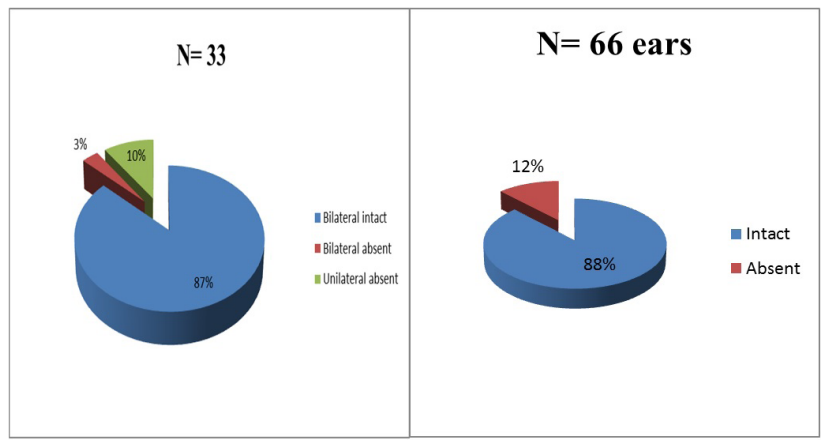

Fig. 1: VEMP results in children with ANSD.

The total number of children was 35 means 70 ears, we mention in the study only 66 ears as the remaining 4 ears were for children having cochlear implantation. The VEMP was absent in those ears, but their results were not included in the current study due to the possible damaging effect of cochlear implantation surgery on the VEMP

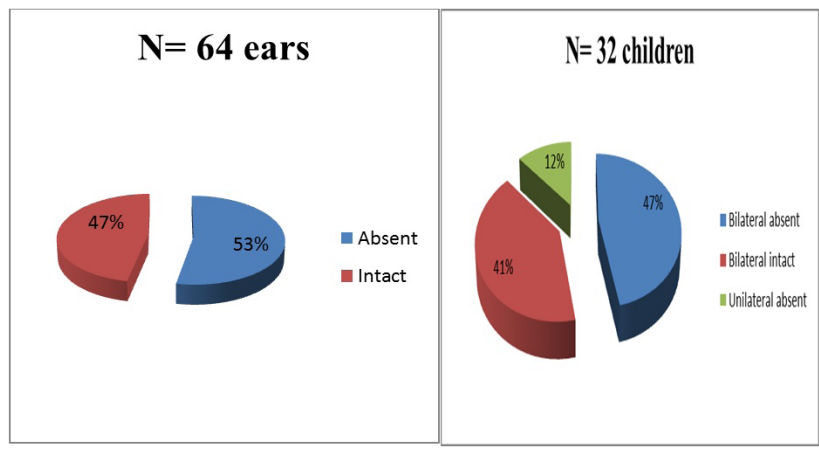

Fig. 2: VEMP results in children with SNHL

There was a significant difference between the ANSD group and non-neuropathy SNHL group as regards the presence versus absence of VEMP as revealed from Chi-square. Chi-square value equal to $38.7 ; P$-value equal .000 (Table 1). However, there is a surprising result that when VEMP was present in ANSD, there is no statistically significant difference between it and VEMP in SNHL group in any parameter as revealed from the (Table 2-5). 
Table 1: Chi-square test for the presence versus absence of VEMP in ANSD and the SNHL groups.

\begin{tabular}{cccc}
\hline & & $\begin{array}{c}\text { Chi-square } \\
\text { value }\end{array}$ & P value \\
\hline $\begin{array}{c}\text { Intact VEMP } \\
\text { in ANSD }\end{array}$ & $58 \quad(88 \%)$ & & \\
$\begin{array}{c}\text { Absent VEMP } \\
\text { in ANSD }\end{array}$ & $8(12 \%)$ & & \\
$\begin{array}{c}\text { Intact VEMP } \\
\text { in SNHL }\end{array}$ & $30 \quad(47 \%)$ & 38.7 & .000 \\
$\begin{array}{c}\text { Absent VEMP } \\
\text { in SNHL }\end{array}$ & $34(53 \%)$ & & \\
\hline
\end{tabular}

Table 2: Comparison among ANSD, SNHL and control groups as regards the corrected P1-N1 amplitude using the ANOVA test

\begin{tabular}{cccccc}
\hline $\begin{array}{c}\text { P1 N1 } \\
\text { amplitude }\end{array}$ & Mean & SD & Range & F & Pvalue \\
\hline Control group & $10.7 \mu \mathrm{v}$ & $5.1 \mathrm{uv}$ & $4.5-24.4 \mathrm{uv}$ & 1.896 & .155 \\
ANSD & $9.5 \mu \mathrm{v}$ & $10.15 \mathrm{uv}$ & $4.2-21.6 \mathrm{uv}$ & & \\
SNHL & $9.2 \mu \mathrm{v}$ & $4.5 \mathrm{uv}$ & $3.8-21.6 \mathrm{uv}$ & & \\
\hline
\end{tabular}

Table 3: Comparison among ANSD, SNHL and control groups as regards the asymmetry ratio using the ANOVA Test

\begin{tabular}{cccccc}
\hline $\begin{array}{c}\text { Asymmetry } \\
\text { ratio }\end{array}$ & Mean & SD & Range & F & Pvalue \\
\hline Control group & $20.4 \%$ & $11 \%$ & $7-47 \%$ & .400 & .672 \\
ANSD & $19.9 \%$ & $14 \%$ & $2 \%-52 \%$ & & \\
SNHL & $19.6 \%$ & $11 \%$ & $4 \%-39 \%$ & & \\
\hline
\end{tabular}

Table 4: Comparison among ANSD, SNHL and control groups as regards the P1 latency using the ANOVA Test

\begin{tabular}{cccccc}
\hline P1 latency & Mean & SD & Range & F & Pvalue \\
\hline Control group & $13.4 \mathrm{~ms}$ & $1.1 \mathrm{~ms}$ & $11.6-17.4 \mathrm{~ms}$ & 1.711 & .185 \\
ANSD & $14.2 \mathrm{~ms}$ & $1.3 \mathrm{~ms}$ & $11.2-17 \mathrm{~ms}$ & & \\
SNHL & $13.9 \mathrm{~ms}$ & $14.4 \mathrm{~ms}$ & $11.4-19.6 \mathrm{~ms}$ & & \\
\hline
\end{tabular}

Table 5: Comparison among ANSD, SNHL and control groups as regards the N1 latency using the ANOVA Test

\begin{tabular}{cccccc}
\hline N1 latency & Mean & SD & Range & F & P value \\
\hline Control group & $18.3 \mathrm{~ms}$ & $1.5 \mathrm{~ms}$ & $16.2-22.6 \mathrm{~ms}$ & 1.664 & .194 \\
ANSD & $19.2 \mathrm{~ms}$ & $1.9 \mathrm{~ms}$ & $16-248 \mathrm{~ms}$ & & \\
SNHL & $18.9 \mathrm{~ms}$ & $2 \mathrm{~ms}$ & $16.4-24 \mathrm{~ms}$ & & \\
\hline
\end{tabular}

Another surprising result that neither any of the risk factors as positive family history\& consanguinity nor the other neonatal\& postnatal history affecting VEMP presence or absence or VEMP parameters in the group of ANSD as revealed from in (Table 6-8). They were showing that there was no statistically significant difference between ANSD children with the risk factor and those without risk factor as regards difference in VEMP parameters\& VEMP presence\& $\mathrm{P} 1-\mathrm{N} 1$ amplitude\& asymmetric ratio. Also, there is no statistically significant difference in P1 latency\& N1 latency and P1-N1 interaural latency difference. The only factor which showing an effect on VEMP is gender as shown in the (Table 9).

Table 6: The Independent Sample Median test comparing ANSD children with risk factors to ANSD children without risk factors as regard VEMP parameters

\begin{tabular}{cccc}
\hline Median & $\begin{array}{c}\text { With risk factors } \\
\text { (Median) }\end{array}$ & $\begin{array}{c}\text { Without risk factors } \\
\text { (Median) }\end{array}$ & P value \\
\hline P1-N1 amplitude & $12.2 \mu \mathrm{v}$ & $9.0 \mu \mathrm{v}$ & .077 \\
P1 latency & $14.1 \mathrm{~ms}$ & $14.2 \mathrm{~ms}$ & .895 \\
N1 latency & $19.8 \mathrm{~ms}$ & $18.6 \mathrm{~ms}$ & .111 \\
$\begin{array}{c}\text { P1-N1 } \\
\text { amplitude AR }\end{array}$ & $21 \%$ & $23 \%$ & .640 \\
\hline
\end{tabular}

Table 7: History of parental consanguinity in children with ANSD

\begin{tabular}{lcc}
\hline Category & Number & P value \\
\hline $\begin{array}{l}\text { Children with an intact VEMP response } \\
\text { and +ve history of consanguinity }\end{array}$ & $16(57 \%)$ & \\
$\begin{array}{l}\text { Children with an intact VEMP response } \\
\text { and -ve history of consanguinity }\end{array}$ & $12(43 \%)$ & \\
$\begin{array}{l}\text { Children with absent VEMP response } \\
\text { and +ve history of consanguinity }\end{array}$ & $3(43 \%)$ & .077 \\
$\begin{array}{l}\text { Children with absent VEMP response } \\
\text { and -ve history of consanguinity }\end{array}$ & $4(57 \%)$ & \\
\hline
\end{tabular}

Table 8: Family history of SNHL in children with ANSD using the Binomial test

\begin{tabular}{|c|c|c|}
\hline Category & $\begin{array}{c}\text { Number and } \\
\text { percentage of children }\end{array}$ & $P$ value \\
\hline $\begin{array}{l}\text { Children with an intact VEMP } \\
\text { response and +ve family history }\end{array}$ & $14(50 \%)$ & \multirow{4}{*}{0.85} \\
\hline $\begin{array}{l}\text { Children with an intact VEMP } \\
\text { response and -ve family history }\end{array}$ & $14(50 \%)$ & \\
\hline $\begin{array}{l}\text { Children with absent VEMP } \\
\text { response and +ve family history }\end{array}$ & $3(43 \%)$ & \\
\hline $\begin{array}{l}\text { Children with absent VEMP } \\
\text { response and -ve family history }\end{array}$ & $4(57 \%)$ & \\
\hline
\end{tabular}

Table 9: Gender distribution of children with ANSD with intact and absent VEMP

\begin{tabular}{lcc}
\hline Category & $\begin{array}{c}\text { Number and } \\
\text { percentage of children }\end{array}$ & P value \\
\hline $\begin{array}{l}\text { Male with an intact } \\
\text { VEMP response }\end{array}$ & $11(31 \%)$ & \\
$\begin{array}{l}\text { Female with an intact } \\
\text { VEMP response }\end{array}$ & $17(49 \%)$ & 0.021 \\
Male with absent & $7(20 \%)$ & \\
VEMP rejoinder & 0 & \\
Female with absent & \\
VEMP response & & \\
\hline
\end{tabular}




\section{DISCUSSION}

For the best of our knowledge, comparable results to the current results are very scanty as the vast majority of ANSD patients included in the previous studies were adults or older children with post-lingual onset ${ }^{[11-12]}$. Only one study ${ }^{[5]}$ reported VEMP in children with pre-lingual onset but in three children, only. Two out of the three children had abnormal VEMP results. In the current work, VEMP findings in young ANSD children with pre-lingual onset were thoroughly described in appreciably large sample size ( 35 children). The youngest child participated in the current study had 3.3 years, whereas The mean age of children was eight years.

In the current study, more than $50 \%$ of ears the SNHL children had an absent VEMP response which is matched with Said (7). However, more than $85 \%$ of the ears of children with ANSD have intact VEMP. This reflecting that there are different sits of pathology in ASND and nonneuropathy SNNHL.

It is not clear why the saccule is affected in some children in the group of SNHL and preserved in the others. Current results showed that there was no significant difference between ANSD children who had absent VEMP and those who had intact VEMP. No significant differences between the two categories as regards age of children, the presence of family history of hearing loss, the presence of consanguinity, the degree of hearing loss, or DPOAE results. The only significant difference was in the gender distribution. Children with absent VEMP response were all males, and none of the female children had an absent VEMP. Genetic origin of X-linked inheritance may explain such finding. Wang et al. ${ }^{[13]}$ results of ANSD patients with $\mathrm{X}$-linked recessive inheritance pattern. They identified a region shared by five affected males and four females carriers that was located between markers DXS1220 and DXS8084 of the X chromosome (region - Xq23-27.3) and named this locus as auditory neuropathy $X$ linked recessive locus 1(AUNX1). Further, they proposed that the ANSD in this family is due to demyelination and axonal loss of the auditory nerve. Similarly, demyelination and axonal loss of the inferior vestibular nerve can explain the absence of VEMP response found in some ANSD male children in the current study.

There was no statistically significant difference between ANSD children with risk factors and those without risk factors as regards the presence or absence of VEMP response. Furthermore, there was no statistically significant difference between the two categories as regards VEMP morphology, P1-N1 amplitude, AR, P1 latency, or N1 latency. All these results were reflecting that the vestibular system more resistant to neuropathy than the auditory system also the vestibular system more resistant to risk factors, even hyperbilubirimina than the auditory system.

There is future ongoing research to examine the remaining parts of the vestibular system in ANSD. As to evaluate the utricle and superior vestibular nerve through the oVEMP testing ${ }^{[14]}$ and the three semicircular canals can be assessed through video head thrust testing ${ }^{[15]}$. All this research may help in better understanding and better management of ANSD.

\section{CONFLICT OF INTEREST}

There are no conflict of interest

\section{REFERENCES}

1. J.S. Lee, B. McPherson, K.C. Yuen, L.L. Wong, Screening for auditory neuropathy in a school for hearing-impaired children, Int. J. Pediatr. Otorhinolaryngol. 61 (2001) 39-46.

2. Y.S. Sininger, Identification of auditory neuropathy in infants and children, Sem. Hear. 23 (2002) 193-200.

3. P.A. Rea, W.P. Gibson, Evidence for surviving outer hair cell function in congenitally deaf ears. Laryngoscope 113 (2003) 230-234.

4. I. Bielecki, A. Horbulewiczt, T. Wolan, Prevalence and risk factors for auditory neuropathy spectrum disorder in hearing loss, Int. J. Pediatr. Otorhinolaryngol. 76 (2012) 1668-1670.

5. Akdogan O1, Selcuk A, Ozcan I, Dere H., Vestibular nerve functions in children with auditory neuropathy, Int J Pediatr Otorhinolaryngol. 72 (3) (2008) 415-9.

6. Zhou G1, Kenna MA, Stevens K, Licameli G. Assessment of saccular function in children with the sensorineural hearing loss. Arch Otolaryngol Head Neck Surg. 135(1) (2009) 40-4.

7. Eman Abdel-Fattah Said, Vestibular assessment in children with sensorineural hearing loss using both electronystagmography and vestibularevoked myogenic potential, Egyptian journal of otolaryngology. 30 (2014) 43-52.

8. Satbir Singh *, Rohit Kumar Gupta, Prawin Kumar. Vestibular evoked myogenic potentials in children with the sensorineural hearing loss. International Journal of Pediatric Otorhinolaryngology. 76 (2012) 1308-1311. 
9. Colebatch JGHalmagyi GMSkuse NF, Myogenic potentials generated by a click-evoked vestibulocollic reflex. J Neurol Neurosurg Psychiatry. 57 (2) (1994) 190- 197.

10. Gary P. Jacobson, Neil T. Shepard. Balance Function Assessment and Management. Second Edition. (2008).

11. Sujeet KS1, Niraj KS1, Animesh B1, Rajeshwari G2, Sharanya R1, Cervical vestibular evoked myogenic potentials, J Vestib Res. 24 (4) (2014) 313-23.

12. K. Sheykholeslami, S. Schmerber, M. Habiby Kermany, K. Kaga, Sacculo colic pathway dysfunction accompanying auditory neuropathy, Acta Otolaryngol. 125 (7) (2005) 786-791.
13. Q. Wang, Q.Z. Li, S.Q. Rao, H. Lee, X.S. Huang, W.Y. Yang, et al., AUNX1, a novel locus responsible for $\mathrm{X}$ linked recessive auditory and peripheral neuropathy, maps to Xq23-27.3, J. Med. Genet. 43 (7) (2006) e 33.

14. Rossengren SM, Govender S, Colebatch JG Ocular\& cervical vestibular evoked myogenic potentials produced by air bone-conducted stimuli: comparative properties and effects of age. Clin Neurophysiol. 122 (11) (2011) 2282-9.

15. Zhang Y, Chen S, Zhong Z, Chen L, Wu Y, Zhao G, Liu Y. Preliminary application of video-head impulse test in the diagnosis of vertigo. Lin Chung Er Bi Yan Hou Tou Wai Ke Za Zhi. 29 (12) (2015) 1053-8. 